\title{
El turismo comunitario como fuente económica en la provincia del Guayas
}

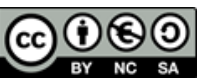

Community tourism as an economic source in the province of Guayas

Yolanda Tatiana Carrasco Ruano. ${ }^{1}$

Recibido: 15-07-2019 / Revisado: 17-08-2019 /Aceptado: 21-09-2019/ Publicado: 05-10-2019

Resumen. $\quad$ DOI: $\underline{\text { https://doi.org/10.33262/concienciadigital.v2i4.933 }}$

El turismo comunitario se presenta como una alternativa a los tradicionales destinos turísticos, esta forma de turismo nos permite mayor contacto con la comunidad y conseguir nuevas experiencias para los viajeros a nivel mundial.

El turismo consiste en los viajes y estancias que realizan personas en lugares distintos a su entorno habitual (al menos durante una noche y como máximo 365 días), por ocio, negocios u otros motivos. Si no se realiza pernoctación, se consideran excursionistas.

El turismo comunitario en el país es una herramienta para incrementar el turismo, como un recurso importante para disminuir la pobreza en los sectores rurales, los cuales han sido beneficiados con bondades naturales que el hombre busca ver, tales como ríos, lagunas, montañas, cascadas, animales, entre otros. Este tipo de turismo se ha vuelto una herramienta dentro del Ecuador, porque muchos lugares no quieren perder su entorno natural y cultura, hay turistas que buscan un lugar donde puedan estar en contacto con la naturaleza. Las comunidades deben trabajar en conjunto para que la visita de las personas se haga muy amena y regresen.

La geografía y la naturaleza ecuatoriana poseen una enorme diversidad de climas y paisajes, tanto de flora como de fauna, el ser humano y la sociedad que habita en medio de estas maravillas, han prosperado en un conjunto ricamente variado de culturas, lenguas, costumbres y cosmovisiones, resultando el país como una amalgama de

\footnotetext{
${ }^{1}$ Ciencia digital, Ambato, Ecuador, tcarrascor@yahoo.com
} 
muchos componentes, siendo así una oportunidad para compartir talentos, destrezas y formas de vida, y así haciendo turismo comunitario.

Palabras claves: Turismo, Economía, Comunidad, Variedad, Sostenibilidad

\section{Abstract.}

Community based tourism is presented as an alternative to the traditional tourist destinations, this form of tourism allows us greater contact with the community and get new experiences for travelers worldwide. The tourism consists of the trips and stays that made people in places other than their usual environment (at least for one night and a maximum of 365 days), for leisure, business or other reasons. If this is not done overnight, are considered to be hikers.

Community based tourism in the country is a tool to increase tourism, as an important resource to reduce poverty in the rural sectors, which have been benefited with natural goodness that man seeks to see, such as rivers, lagoons, mountains, waterfalls, animals, among others. This type of tourism has become a tool within Ecuador, because many places don't want to lose its natural environment and culture, there are tourists who are looking for a place where they can get in touch with nature. The communities must work together for the visit of the people to become very entertaining and return.

Geography and Nature in Ecuador have an enormous diversity of climates and landscapes, of flora and fauna, the human being and society that dwells in the midst of these wonders, have prospered in a richly varied set of cultures, languages, customs and worldviews, resulting in the country as an amalgam of many components, thus being an opportunity to share talents, skills and ways of life, and in so doing community-based tourism.

Keywords: Tourism, Economy, Community, Variety, Sustainability.

\section{Introducción.}

\section{Contraste de autores}

Según los autores Contreras A. 2015, Espinoza O. 2015 \& Loor K. 2012, el turismo comunitario es un buen uso para aprovechar los recurso naturales que ofrece la provincia del Guayas, ya que esto deja grandes ingresos en las comunidades que se encuentran estos atractivos turísticos. La idea del turismo comunitario como fuente de desarrollo económico y social en la provincia del Guayas es una idea acertada ya que con el propósito de viaje de los visitantes extranjeros hay más ingresos para las comunidades. 


\section{Criterio Personal}

El turismo comunitario es importante ya que hay una organización de las comunidades, barrios, grupos étnicos o emprendedores que sepan potenciar las diversas áreas ecológicas, naturales, urbanas que posee está ciudad, o comenzar a emprender proyectos productivos comunitarios basados en la demanda turística, de esta manera se benefician los miembros de las comunidades locales, ya que se convierte en una alternativa económica, directa e indirectamente al turismo comunitario ya que contribuye a este gran proyecto nacional de convertir al turismo en el primer ingreso no petrolero del país, en el gran Guayas ya que está logrando, con muchos inconvenientes y limitaciones pero de apoco se va desarrollando en estos últimos tiempos en la mayoría de los pueblos del Ecuador se ha venido desarrollando lo que es el Turismo Comunitario, con la finalidad de obtener mayores beneficios para la zona a través de esta actividad; pero, en muchas ocasiones esto no resulta así, ya sea por la falta de promoción que tiene un determinado destino o el poco cuidado que se le ha dado a sus atractivos por parte de sus pobladores, debido a que estos en innumerables ocasiones no han sido capacitados correctamente para el uso adecuado de sus recursos tanto naturales como culturales.

Es por esto, que en esta investigación el objetivo principal será: Sentar las bases para el desarrollo del Turismo Comunitario en la zona de Dos Mangas y Barcelona, rescatando en estas comunidades la riqueza turística, cultural y natural. Es decir proporcionar a estas comunidades las herramientas necesarias como lo son los talleres de capacitación para que de esta manera ellos puedan cuidar, mantener y explotar sus recursos satisfactoriamente. A lo largo de nuestro trabajo hemos estado en contacto con los pobladores de ambas comunidades quienes nos han proporcionado toda la ayuda e información que hemos requerido para el desarrollo del mismo, también hemos realizado encuestas para saber hasta qué grado ellos están dispuestos a participar en la actividad turística, así mismo hemos tomado de ejemplo los proyectos que se están llevando a cabo en otras comunidades para de esta manera tener una visión más amplia de lo que es el Turismo Comunitario.

En los últimos años se observa un cambio en los gustos de los viajeros que consiste en conocer nuevos destinos, realizar distintas actividades y buscar propuestas relacionadas en ciertos casos con las costumbres locales, con lo étnico y el origen de determinadas culturas .En este sentido se busca experimentar con la diversidad cultural como forma de enriquecer a los propios turistas ,todo ellos ha permitido que determinados lugares fueran de los tradicionales circuitos turísticos que puedan posibilitar la creación de los productos turísticos y así poder arbitrar nuevas oportunidades que generan riqueza en determinadas áreas a través de estrategias de participación de la mayoría de la población. 


\section{Contraste de autores}

Según los autores Gutiérrez S. 2013, Luna K. 2015 \& Vargas H. 2013, las ingresos económicos que deja el turismo comunitario son muy útiles ya que con esto se puede hacer que los habitantes tengan un buen descanso en un buen estado los sectores donde se practica este tipo de actividades son en las comunidades ya que puede recrear su mente y su espíritu.

\section{Criterio Personal}

El turismo comunitario permite a los habitantes de la región dada convertirse en guías turísticos y prestar servicios a los visitantes que reciben. Ofrece al propio tiempo a las comunidades la oportunidad de jugar un papel en la generación del movimiento de los elementos de producción e intercambio de bienes y servicios en la zona en cuestión, hoy en día, el turismo comunitario sigue en constante evolución, no solo en la provincia del Guayas generando una participación más activa del turista dentro de los hábitos y costumbres de un grupo social o localidad en particular.

Es por esto, que en estas comunidades la riqueza turística, cultural y natural proporciona a estas comunidades las herramientas necesarias como lo son los talleres de capacitación para que de esta manera ellos puedan cuidar, mantener y explotar sus recursos satisfactoriamente. A lo largo de nuestro trabajo hemos estado en contacto con los pobladores de ambas comunidades quienes nos han proporcionado toda la ayuda e información que hemos requerido para el desarrollo del mismo, también hemos realizado encuestas para saber hasta qué grado ellos están dispuestos a participar en la actividad turística, así mismo hemos tomado de ejemplo los proyectos que se están llevando a cabo en otras comunidades para de esta manera tener una visión más amplia de lo que es el Turismo Comunitario.

El turismo comunitario es un tipo de actividad turística que se enfoca en la creación de negocios turísticos que deben ser manejados íntegramente o en su mayoría por la comunidad anfitriona y donde los beneficios que se obtengan de la misma deben ser repartidos con equidad entre toda la colectividad.

En Ecuador, el turismo comunitario se ha venido gestando desde la década de los años 70, cuando asociaciones campesinas y dirigentes indígenas, empezaron a buscar reconocimiento político, consolidaron diversas acciones que, finalmente, revalorizaron la cultura indígena y sus características principales. En la actualidad, el Ministerio de Turismo de Ecuador presenta, entre sus productos principales el programa "Consolida Turismo Comunitario", cuyo objetivo principal es mejorar el producto turístico comunitario mediante el apoyo directo del Ministerio de Turismo a las comunidades que desarrollan actividades turísticas, y entre sus fines se encuentra el apoyo técnico, de capacitación y promoción de los nuevos negocios turísticos comunitarios que se vayan formando. 


\section{Contraste de autores}

Según los autores Villon C. 2012, Holguin R. 2015 \& Viteri M. 2010, el turismo comunitario Es un tipo de turismo caracterizado por la intervención de la comunidad local respecto al turismo, que se realiza en su sector, de esta forma los beneficios quedan en manos de miembros que conforman estas comunidades ya sean de tipo campesino, indígenas, mestizas, etc.

\section{Criterio Personal}

Los beneficios para las comunidades que participan en el desarrollo del turismo son varios, como la reducción de la pobreza y la generación de fuentes de empleo. A ello se suma que este enfoque descentralizar la industria del turismo mediante la transferencia de la toma de decisiones a las comunidades, contribuyendo así al desarrollo de productos turísticos regionales, además de conducir al fortalecimiento de las comunidades locales, al tiempo que actúa como un estímulo para la producción de productos locales, como la gastronomía, entre otros.

Si bien es cierto que existen experiencias en turismo comunitario en Ecuador que se han trabajado y estudiado desde hace algunos años (Bacci, M.E., y Mujica, E. Turismo rural en el Ángel, Carchi, Ecuador: perspectivas y posibilidades. Caracas, 2001; ver también el caso de Salinas de Guaranda), lo cierto es que la mayoría de estas experiencias se han realizado en la sierra ecuatoriana, mientras que en la región costa, son pocos los emprendimientos de este tipo de modalidad turística. Sin embargo, existen comunidades no muy conocidas que están buscando oportunidades de desarrollo y formación de nuevos negocios. Una de ellas es la comuna "Cerrito de los Morreños", ubicada en el cantón Guayaquil, Provincia del Guayas, cuyos habitantes se encuentran interesados en el desarrollo de la actividad turística en su localidad. El presente caso de estudio muestra las oportunidades de desarrollo turístico de la mencionada comunidad, sus características principales y una evaluación general del potencial que posee para dedicarse a la actividad de turismo comunitario.

Realizar turismo en las comunidades y pueblos del Ecuador, se traduce al resguardo de sus territorios de vida y a la visibilización de sus culturas, de los pueblos y nacionalidades, los movimientos indígenas y afro ecuatorianos, con el respaldo de las autoridades locales y autoridades nacionales, más el acompañamiento del Consejo de Desarrollo de los Pueblos y Nacionalidades del Ecuador, los cuales han propuesto en discusión la necesidad y el derecho al reconocimiento y legalización de la actividad comunitaria en el turismo.

Existen varios ejemplos de turismo comunitario en el país que están dando magníficos resultados como por ejemplo Agua blanca, Salango, Yunguilla, comunidad de Bilsa, Muisne, Comunidad de Pajiaza (Manglar alto), entre otras, es por eso que se quiere abrir la posibilidad de un turismo Comunitario en Nanegalito intentando resaltar sus atractivos naturales, con el 
desarrollo de nuevos productos turísticos que serán creados y así se contribuirá al desarrollo de esta comunidad. Según entiendo todo plan comunitario tiene como propósito que sus recursos naturales se conviertan importantes dentro del área de turismo, con el desarrollo mejorar la estructura y buscar su potencial para la promoción de estos.

\section{Contraste de autores}

Según los autores Barrera K. 2014, Ormaza P. 2013 \& Ulloa J. 2014, las comunidades en las que se practica el turismo comunitario es una de las fuentes de ingreso más útiles en los sectores ya que ese dinero que entra ellos pueden mantener limpias las zonas donde los turistas visitan que permita encaminarse a alcanzar unos objetivos comunes y predeterminados para mejorar las condiciones económicas, sociales y culturales de las comunidades, y cuyos resultados puedan ser evaluados de forma continua.

\section{Criterio Personal}

El turismo puede ser una forma de diversificar las actividades económicas de una comunidad, pero no la única vía, ni necesariamente la más importante, y mucho menos una opción para todo el país. Es por ello que es especialmente importante que los planes de desarrollo comunitario no estén centrados en un solo rubro, ya sea el turismo o cualquier otro, sino en la diversidad y complementariedad de acciones que pueden favorecer al conjunto de su población y que, a su vez, traten de evitar los riesgos de generar nuevos procesos de diferenciación y agudización de las desigualdades

El turismo es una actividad que se ha establecido como una interesante estrategia para el desarrollo de las comunidades; sin embargo se considera que para que el turismo sea sostenible es necesario un mínimo de organización social para que los diferentes sujetos sociales puedan ser los protagonistas de las transformaciones de sus propios lugares, los pueblos indígenas y sus comunidades gozan del derecho a decidir sus propias prioridades en lo que atañe al proceso de desarrollo, además. Para lograr un desarrollo sostenido y equitativo, los gobiernos deben fortalecer las actividades de la economía tradicional y adoptar medidas encaminadas a allanar las dificultades que experimentan dichos pueblos al afrontar nuevas condiciones de vida y de trabajo.

El turismo comunitario "es una actividad que se fundamenta en la creación de productos turísticos bajo el principio básico de la necesaria participación de la comunidad local". Relacionando este criterio basado en la comunidad también se pude mencionar que el turismo comunitario "pretende reducir el impacto negativo y reforzar los impactos positivos del turismo en la naturaleza", asimismo está catalogado "como aquel tipo de turismo que se desarrolla en áreas naturales y tiene como objetivo la conservación de las zonas naturales y el desarrollo de las comunidades locales". 
Tomando como referencia el lugar de estudio del presente trabajo de titulación se debe mencionar a la (Federación Plurinacional de Turismo Comunitario, s.f.), quienes impone como concepto de turismo comunitario lo siguiente: "Relación de la comunidad con los visitantes desde una perspectiva intercultural en el desarrollo de viajes organizados con la participación consensuada de sus 12 miembros, garantizando el manejo adecuado de los recursos naturales, la valoración de sus patrimonios, los derechos culturales y territoriales de las nacionalidades y pueblos para la distribución equitativa de los beneficios generados.

El desarrollo comunitario es un método de intervención que incorpora a todos los agentes que conforman la comunidad, estableciendo procesos de participación y articulación entre la población y las instituciones (fundamentalmente las estructuras municipales, aunque no únicamente) que, potenciando un proceso pedagógico, y las capacidades participativas de los actores y de las estructuras mediadoras (técnicos, profesionales y entidades sociales), permita encaminarse a alcanzar unos objetivos comunes y predeterminados para mejorar las condiciones económicas, sociales y culturales de las comunidades, y cuyos resultados puedan ser evaluados de forma continua.

Como visión estratégica para el desarrollo económico y social de los ciudadanos Guayaquileños, se ha planteado la implementación el turismo comunitario. A nivel nacional, regional y local, el turismo se ha convertido en una prioridad que requiere recursos e inversión, para poder desempeñar el rol que este atribuye a ciertos países en aspecto socioeconómico como fuente de divisas, inclusión social, empleo, entre otros. La creación de una oferta turística basada en principios del turismo comunitario ha llegado a tener buenos resultados en ciertos lugares de Guayaquil donde se lo ha puesto en práctica, pero la escasa inversión e incentivo del municipio local pone en evidencia la poca expansión y el lento crecimiento de esta tendencia turística. La idea del turismo comunitario como fuente de desarrollo económico y social en una ciudad tan grande como Guayaquil que actualmente (año 2015) tiene 2560505 habitantes, es una idea acertada ya que como pudimos comprobar que el propósito de viaje de los visitantes extranjeros está liderada por las vacaciones con un $43 \%$ y dentro de la subdivisión de vacaciones el turismo cultural es la primera elección con el $12 \%$.

Para lograr crear una tendencia positiva hacia el turismo comunitario es importante la organización de las comunidades, barrios, grupos étnicos o emprendedores que sepan potenciar las diversas áreas ecológicas, naturales, urbanas que posee está ciudad, o comenzar a emprender proyectos productivos comunitarios basados en la demanda turística. De esta manera se benefician los miembros de las comunidades locales, ya que se convierte en una alternativa económica. Directa e indirectamente el turismo comunitario contribuye a este gran proyecto nacional de convertir al turismo en el primer ingreso no petrolero del país, en Guayaquil se está logrando, con muchos inconvenientes y limitaciones pero de apoco se va desarrollando. 


\section{Conclusiones}

- El turismo comunitario se está desarrollando en diferentes zonas del mundo como consecuencias de la búsqueda que realizan los viajeros para descubrir nuevas alternativas a los viajes tradicionales, lo cual está posibilitando la creación de determinados destinos que permiten a las propias comunidades que generan riqueza con la actividad tradicional basada fundamentalmente en las tareas agrícolas, ganaderas y pesqueras. También es valorado de forma muy positiva por la comunidad ya que así se puede generar beneficios y a la vez crean fuentes de trabajo.

- Se puede decir que la provincia del Guayas se ha destacado principalmente por el trabajo que realizan con paja toquilla que actualmente es reconocida en otras provincias del país y la comunidad de Dos Mangas lo ha hecho en la fabricación de artesanías en tagua, que son vendidas a la misma Fundación y a la ciudad de Guayaquil.

- El acercamiento con otros grupos poblacionales que en iguales circunstancias lograron cambios importantes que mejoraron su calidad de vida, es un elemento motivante, es importante mantener o replicar dicha experiencia, ya que al parecer tiene el efecto esperado.

- El turismo es un sistema dinámico que permite el mejoramiento de la calidad de vida de una población específica, por lo tanto se desarrolló e inclusión turística de la Comunidad Shuar del Río Limón en el cantón Bucay Provincia del Guayas.

- Las diferentes teorías turísticas que han permitido identificar las estrategias que permitan el desarrollo del turismo comunitario en el sector de Río Limón de la provincia del Guayas, además de la aplicación de conceptos fundamentados en la tendencia actual de la sostenibilidad.

\section{Referencias bibliográficas.}

Alvarez, R. (2013). El turismo comunitario en distintas partes del pais. Quito, Ecuador: El Comercio.

Barrera, K. (2014). El turismo sostenible en la económia del pais. Quito, Ecuador.

Cabrera, Y. (2012). Las fuentes económicas del turismo comunitario en la provincia del Guayas. Guayaquil, Ecuador.

Contreras, A. (2015). El turismo comunitario en los pueblos del Ecuador. (http://www.revistaespacios.com/a18v39n07/18390728.html, Ed.) Quito, Ecuador.

Espinoza, O. (2015). El turismo comunitario en los resintos Dos Mangas y Barcelona del cantón Buacy. Guayaquil, Ecuador. 
Gutierrez, S. (2013). El turismo comunitario en la economia de la provincia. Guayaquil, Ecuador.

Holguin, R. (2015). Las autoridades que conforman el turismo comunitario. Cuenca, Ecuador:

http://www.guayas.gob.ec/turismo/dmdocuments/consultoria/Cerrito_Morrenos.pdf.

Loor, K. (2012). Comunidades que prestan turismo comunitario en la provincia del Guayas. . Daule, Ecuador.

Luna, K. (2015). El turismo comunitario. Guayaquil, Ecuador.

Ormaza, P. (2013). Actividades fundamentales del turismo comunitario. Guayaquil, Ecuador.:

http://repositorio.ug.edu.ec/bitstream/redug/3482/1/Urgil\%C3\%A9s\%20Andrade\%2 0Priscila\%20Bel\%C3\%A9n.pdf.

Rodriguez, K. (2012). Las fuentes turisticas en el cantón Bucay de la provincia del Guayas. Salitre, Ecuador.

Ulloa, J. (2014). El turismo comunitario en convivencia de la biodiversidad. Quito, Ecuador.: El Comercio.

Vargas, H. (2013). El turismo comunitario en conjunto con identidades publicas. Bucay, Ecuador: http://www.revistaespirales.com/index.php/es/article/view/113.

Verdezoto, A. (2015). El turismo comunitario, en la provincia del Guayas. Guayaquil, Ecaudor.

Villon, C. (2012). Distintos tipos de turismo comunitario en el cantón Bucay. Guayaquil, Ecuador.: UNIVERSIDAD CATÓLICA DE SANTIAGO DE GUAYAQUIL.

Viteri, L. (2014). La importancia del turismo comunitario en las comunidades. Lima, Perú.

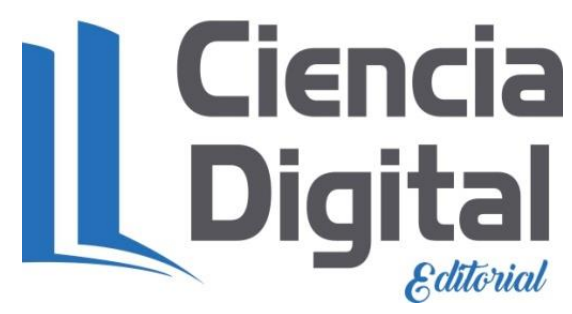


Para citar el artículo indexado.

Carrasco Ruano, Y. (2019). El turismo comunitario como fuente económica en la provincia del Guayas. ConcienciaDigital, 2(4), 56-65.

https://doi.org/10.33262/concienciadigital.v2i4.933

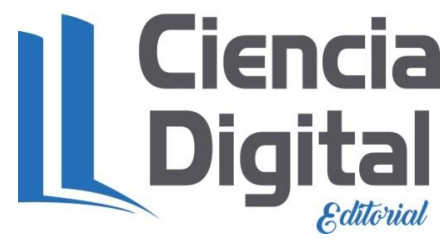

El artículo que se publica es de exclusiva responsabilidad de los autores y no necesariamente reflejan el pensamiento de la Revista Conciencia Digital.

El articulo queda en propiedad de la revista y, por tanto, su publicación parcial y/o total en otro medio tiene que ser autorizado por el director de la Revista Conciencia Digital.

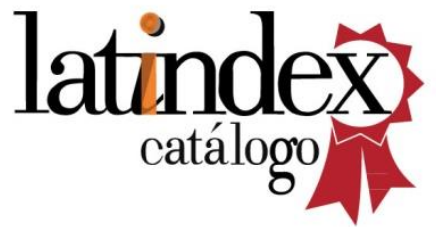

Conciencia

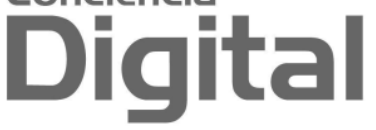

